\title{
Optimisation d'une source d'ultraviolet fonctionnant dans un réacteur de traitement d'eau de consommation
}

\author{
C. Costache Mongrand, J.J. Damelincourt et G. Zissis
}

\author{
Centre de Physique des Plasmas et de leurs Applications de Toulouse, CPAT, Université Paul \\ Sabatier, 118 route de Narbonne, 31062 Toulouse cedex 4, France
}

\begin{abstract}
Résumé : Nous nous sommes attachés à aborder le problème de l'optimisation d'un système de traitement d'eau par rayonnement ultraviolet en examinant et en modélisant successivement les principaux couplages qui interviennent dans un tel système. Un exemple de résultats sur le couplage thermique réacteur- source est présenté. Cette modélisation peut en effet conduire à des solutions qui n'auraient pas été envisagées autrement.
\end{abstract}

\section{INTRODUCTION}

La désinfection de l'eau de consommation par irradiation ultraviolette reste une alternative intéressante à la chloration. Elle est considérée comme très efficace autour d'une longueur d'onde du rayonnement voisine de $265 \mathrm{~nm}$. Ceci explique pourquoi la décharge mercure gaz rare basse pression, caractérisée par une production extrêmement efficace de rayonnement ultraviolet à $253,7 \mathrm{~nm}$, est généralement utilisée pour assurer cette fonction.

Le rendement énergétique et l'efficacité biologique du système d'irradiation ultraviolette dépendent d'assez nombreux paramètres : dimensionnement, remplissage et mode d'alimentation des lampes à décharge, température de l'eau à traiter, transport du rayonnement dans le réacteur, nature des écoulements hydrauliques. Parmi ces paramètres, il nous a paru intéressant d'examiner dans queile mesure l'effet thermique de l'eau à traiter sur la lampe pouvait remettre en cause les choix faits pour définir le remplissage gazeux des sources utilisées.

\section{COUPLAGE DECHARGE-REACTEUR}

L'efficacité des lampes mercure gaz rare basse pression dépend de façon critique de leur température de fonctionnement (température du point le plus froid de la paroi). Généralement, le choix des paramètres de construction est fait pour optimiser la production du rayonnement pour une température du point froid voisine de $40^{\circ} \mathrm{C}$. La température de l'eau dans le réacteur est, en général, comprise entre 5 et $30^{\circ} \mathrm{C}$. Nous pouvons alors utiliser cette source froide pour maintenir la lampe à une température proche de la température optimale de fonctionnement. Cette température optimale dépend également des autres paramètres de la lampe. Nous avons donc cherché, en tenant compte de l'environnement de la lampe, à adapter les paramètres de construction du radiateur ultraviolet pour obtenir une plus forte production de rayonnement ultraviolet tout en conservant un rendement acceptable.

Dans ce réacteur, et c'est la configuration la plus courante, la source de rayonnement ultraviolet est placée dans l'axe d'un tube en quartz qui évite son contact avec l'eau. L'espace entre les deux tubes est rempli d'air à la pression atmosphérique, sans aucune ventilation artificielle. Nous avons considéré que le flux d'air est laminaire. En sachant que la température de l'eau varie entre 5 et $30^{\circ} \mathrm{C}$ et la température maximale de la lampe entre 50 et $60^{\circ} \mathrm{C}$, nous avons pu négliger le transfert thermique par rayonnement. En supposant que la silice laisse passer la totalité du rayonnement ultraviolet produit par la décharge, le dépôt d'énergie sur la paroi n'est dû qu'aux collisions élastiques, $\mathrm{P}_{\mathrm{er}}$. La figure 1 donne le schéma qui sert de support à la modélisation. Le modèle de décharge utilisé est un modèle collisionnel-radiatif basé sur la résolution des équations de conservation de masse et d'énergie [1]. 


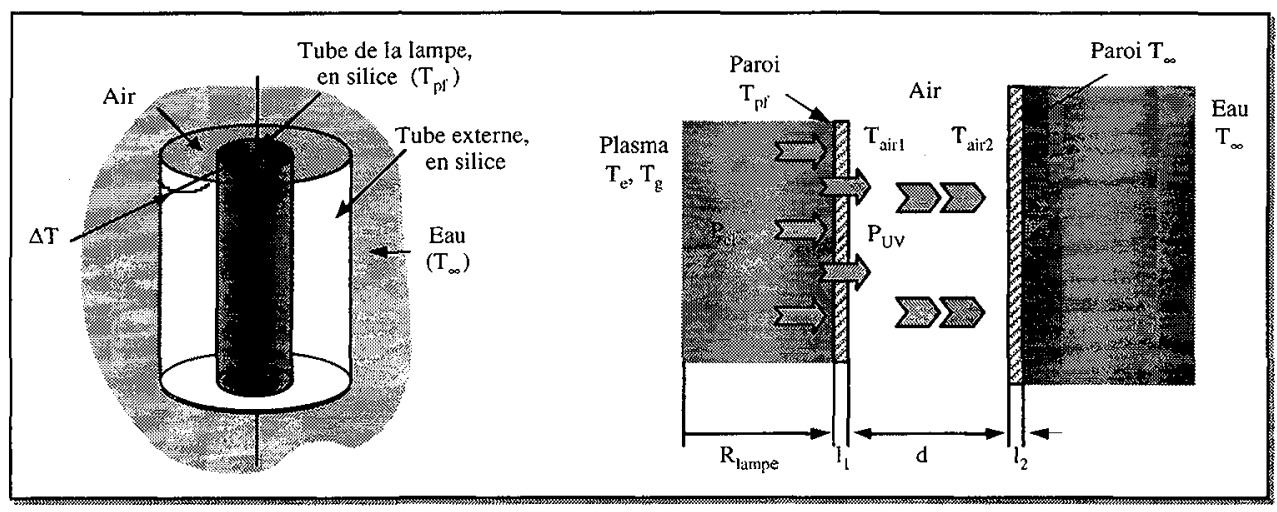

Figure 1 : Configuration utilisée pour le couplage thermique lampe-réacteur (à gauche) ; schéma de modélisation (à droite)

\section{RESULTATS}

Nous avons fait varier plusieurs paramètres caractéristiques de la décharge, nature et pression du gaz rare, courant d'alimentation et nous avons étudié leur influence sur le rayonnement émis. Par exemple, pour une décharge établie dans un tube de diamètre $15 \mathrm{~mm}$ rempli de 1 Torr de Krypton et fonctionnant avec un fort couplage thermique et une température d'eau de $20^{\circ} \mathrm{C}$, le flux ultraviolet passe de $32 \mathrm{~W} / \mathrm{m}$ à $57 \mathrm{~W} / \mathrm{m}$ lorsque le courant est augmenté de 400 à $800 \mathrm{~mA}$. Les rendements ultraviolets correspondants valent respectivement $52 \%$ et $63 \%$. Nous gagnons donc à la fois en puissance rayonnée et en rendement par rapport à une utilisation classique à $400 \mathrm{~mA}$. Le gain en dimensionnement global est aussi intéressant.

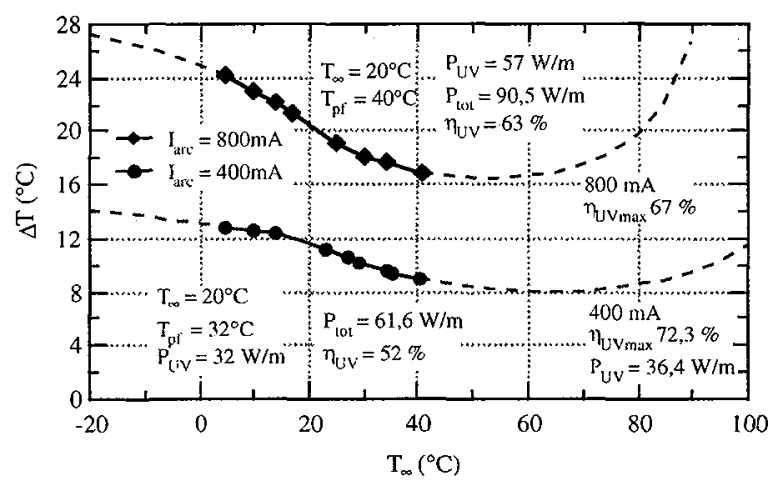

Figure 2: Influence de la température de l'eau sur le bilan global à 400 et $800 \mathrm{~mA}$ ( $\Delta \mathrm{T}$ représente la différence de températures entre l'eau $T_{\infty}$ et la lampe $T_{p f}$ )

\section{CONCLUSION}

Le but de cette étude était de rechercher les paramètres externes optimaux du radiateur ultraviolet dans son environnement. Les calculs nous ont montré que nous obtenions effectivement de meilleurs résultats en adaptant, à l'aide d'une modélisation globale du système, les caractéristiques de construction de la décharge aux propriétés thermiques du système.

\section{Référence}

1. G. Zissis, J.J. Damelincourt, Proc. IEEE Int. Conf. on Plasma Science, p 218, Vancouver, (1993) 